\title{
Molecular Dynamics of Diffusive-Ballistic Heat Conduction in Single-Walled Carbon Nanotubes
}

\author{
Junichiro Shiomi and Shigeo Maruyama* \\ Department of Mechanical Engineering, The University of Tokyo, 7-3-1 Hongo, \\ Bunkyo-ku Tokyo 113-8656, Japan
}

Diffusive-ballistic heat conduction of finite-length single-walled carbon nanotubes has been studied by means of non-equilibrium molecular dynamics simulations. The length-dependence of thermal conductivity is quantified for a range of nanotube-lengths up to a micrometer at room temperature. A gradual transition from nearly pure ballistic to diffusive-ballistic heat conduction was identified from the thermal conductivity profile. In the diffusive-ballistic regime, the profile exhibits power-law length dependence and does not converge even with the tube-length of a micrometer. Furthermore, the diameter dependence of thermal conductivity suggests considerable suppression of phonon scattering effect as the diameter decreases.

KEYWORDS: carbon nanotube, molecular dynamics, thermal conductivity, phonon transport

*E-mail address: maruyama@photon.t.u-tokyo.ac.jp 


\section{Introduction}

The expanding expectations for single-walled carbon nanotubes (SWNTs) include applications for various electrical and thermal devices due to their unique properties. ${ }^{1)}$ SWNTs are expected to possess high thermal conductivity due to their strong carbon bonds and the quasi-one-dimensional structure. ${ }^{2)}$ On considering the actual applications, one of the essential tasks is to characterize the thermal properties not only for thermal devices but also for electrical devices since they determine the affordable amount of electrical current through the system.

Following the thermal conductivity measurements of SWNTs in forms of mats and bundles $^{3)}$, with advances in SWNT synthesis and MEMS techniques, thermal conductivity (or thermal conductance) measurements of individual carbon nanotubes have been recently reported not only for multi-walled carbon nanotubes ${ }^{4,5)}$, but also for SWNTs. ${ }^{6,7)}$ However, thermal property measurements of SWNTs in experiments are still extremely challenging as there are potential uncertainties residing in the technicality for instance related to the contact resistances between thermal reservoirs and an SWNT. Uncertainties also arise in the identification of an isolated SWNT and its diameters. Therefore, reliable theories and numerical simulations are greatly demanded especially to investigate detailed heat conduction characteristics that are not yet accessible in experiments. One of such heat conduction characteristics with a practical importance is the size effect on thermal conductivity. In general, the size-dependence of thermal conductivity appears when the system characteristic length is smaller or comparable to the phonon mean free path. ${ }^{8)}$ For SWNTs, due to the expected long phonon mean free path, the regime of the length effect stretches beyond the realistic length in many applications. The length effect has been demonstrated using molecular dynamics (MD) simulations $^{9,10)}$ and the power-law divergence was discussed with analogy to the low-dimensional models, where the hydrodynamic effect gives rise to the long-time heat flux correlation. ${ }^{11)}$ More recently, the length dependence of thermal conductivity was investigated up to fully diffusive phonon transport regime using a kinetic approach ${ }^{12}$, where the divergence due to long wave-length phonons was shown to disappear with presence of the second order (or higher) 3-phonon scattering processes. The issue of the transition from the pure ballistic to diffusive-ballistic phonon transport has been discussed by modeling the energy transmission based on the ratio of the overall average phonon mean free path to $L .{ }^{13)}$

This paper aims to demonstrate the ballistic diffusive transition of heat conduction in SWNTs at room temperature by calculating thermal conductivity for a range of lengths using non-equilibrium classical MD simulations. MD simulations are capable of handling phonon transport of all the phonon branches, unlike the kinetic approach with relaxation approximations. ${ }^{12)}$ As shown later, this aspect is important for relatively short 
SWNTs with significant ballistic phonon transport, especially at room temperature, where a wide range of phonon branches are populated.

\section{Molecular Dynamics Model}

The carbon-carbon interactions were modeled using Brenner potential ${ }^{14)}$ in a simplified form ${ }^{15)}$ where the total potential energy of the system is expressed as,

$$
E=\sum_{i} \sum_{j(i<j)}\left[V_{R}\left(r_{i j}\right)-B_{i j}^{*} V_{A}\left(r_{i j}\right)\right]
$$

Here, $V_{R}(r)$ and $V_{A}(r)$ are repulsive and attractive force terms, which take a Morse type form with a certain cut-off function. $B^{*}{ }_{i j}$ represents the effect of the bonding order parameters. As for the potential parameters, we employ the set that has been shown to reproduce the linear phonon transport properties with sufficient accuracy. ${ }^{9,10)}$ The velocity Verlet method was adopted to integrate the equation of motion with the time step of 0.5 fs. The application of classical approach is encouraged by the expected dominant contribution on the heat conduction from phonons compared with that from electrons. ${ }^{16,17)}$

On simulating ballistic phonon transport using MD simulations, linear transport properties (group velocity) need to be reproduced with sufficient accuracy. This needs to be satisfied for phonons with a wide range of frequencies as their ballistic transport becomes important for short SWNTs at room temperature. Linear phonon transport properties can be visualized by the dispersion relations, which can be computed from MD simulations by taking the two-dimensional Fourier spectra of the time history of the one-dimensional velocity field along an SWNT. In Fig. 1, the spectra of a $25 \mathrm{~nm}$ (5, 5)-SWNT at $300 \mathrm{~K}$ with the periodic boundary condition is presented together with corresponding phonon density of states (DOS). The phonon energy spectral density is computed as ${ }^{18)}$,

$$
\Phi(\omega, k)=\frac{m}{2} \sum^{p} \sum_{\alpha}^{3}\left|\frac{1}{N} \sum_{n=0}^{N-1}\left[e^{i \frac{n}{N} k} \int v_{\alpha}(n, t) e^{-i \omega t} d t\right]\right|^{2},(\alpha=r, \phi, z),
$$

where $N$ is the number of atoms in the longitudinal $(z)$ direction (the number of unit cells in the nanotube). $p$ and $m$ are the number of atoms per unit cell and the mass of a carbon atom, respectively. The data are discrete due to the finite length of the nanotube and the broadening of the spectral peaks indicates the thermal phonon scattering. The overall feature of dispersion relations obtained from MD simulations agrees with the reported theoretical models ${ }^{1,19)}$, especially well with the mechanical model of Mahan and Jeon ${ }^{19)}$. The phonon DOS $g(\omega)$ can be calculated from $g(\omega) \hbar \omega \eta(\omega)=\sum_{k} \Phi(\omega, k)$, where the equilibrium phonon distribution $\eta(\omega)=k_{B} T / \hbar \omega$ at the classical limit. 
Note, for SWNTs, as the number of phonon branches is determined by the number of atoms in a unit-cell, even armchair (or zigzag) SWNTs whose unit-cell contains fewer atoms than the other structures with similar diameters, the dispersion relation depicts diverse phonon branches, as seen in Fig. 1 for a $(5,5)$ SWNT. There are optical phonon modes with small circumferential wave number and low frequency that have similar dispersion characteristics and heat capacity to the acoustic ones, especially in the intermediate wavevector $(k)$ regime. Although, acoustic modes may still possess the longest mean free path, the contribution of these optical modes is expected to become important when their mean free paths are comparable to $L$.

\section{Thermal Conductivity Calculations}

Thermal conductivity $\lambda$ of an SWNT was measured with non-equilibrium MD simulations. After reaching an isothermal state at $300 \mathrm{~K}$ with the auxiliary velocity scaling control, the temperature controlled layers on both ends of the SWNT were activated to apply a temperature difference of $20 \mathrm{~K}$. Eventually the system converges to a quasi-stationary state with linear temperature gradient. The simulation time ranges within 3-18 ns as the data-convergence time depends on the system size. By calculating the heat flux along the SWNT from the energy budgets of the thermostats, $\lambda$ was calculated through the Fourier's law. The cross-sectional area $A$ of an SWNT was defined using the ring of van der Waals thickness $\pi b d$, where $b=0.34 \mathrm{~nm}$. The validity of the temperature gradient $20 \mathrm{~K}$ for short SWNTs was examined by performing additional simulations for temperature gradient of $10 \mathrm{~K}$ in case of $L=25 \mathrm{~nm}$. The converged value of thermal conductivity was $236 \mathrm{~W} / \mathrm{mK}$ compared to $223 \mathrm{~W} / \mathrm{mK}$ for $20 \mathrm{~K}$ gradient case. Hence, the difference was confirmed to be small enough. The usage of thermal conductivity to express the heat conduction of the current system is arguable due the extensive ballistic heat transport. Furthermore, the definition of the area of an isolated SWNT is rather ambiguous. Although simply expressing the heat conduction with thermal conductance may be more suitable, here we use thermal conductivity for the sake of comparison with previous studies.

On carrying out non-equilibrium MD simulations by locally applying thermostats to a crystal system, the interface between the temperature-controlled part and the rest of the system typically gives rise to a thermal boundary resistance (TBR). A TBR appears due to mismatching of lattice-vibrational spectra of the temperature controlled part and the rest of the system. The mismatching causes reflection of phonons and alters scattering dynamics at the interface. Since a TBR is expected to influence the local non-equilibrium phonon distribution and hence alter the thermal conduction, thermostats and their parameters need to be carefully selected to minimize the TBR.

It is important to state that the TBR effect is not entirely a numerical artifact. For 
instance, in practical use of the lateral heat conduction of SWNTs to promote heat transfer, finite-length SWNTs would be bounded with connections to other materials. In this case, the heat conduction properties would be inevitably altered by TBRs at the connections. Therefore, in fact, it would be more realistic to examine the heat conduction of SWNTs with presence of such interfacial thermal resistances, though formulation of a general case would be difficult since such effects would be strongly case-dependent. In the current study, for the sake of comparison with other reported theoretical works and focusing on studying the intrinsic dynamics, we aim to construct an ideal case by minimizing the TBR effect.

Firstly, the temperature gradient was applied using the phantom technique as in the previous works. ${ }^{9,10)}$ Here, a phantom thermostat consists of a fixed layer and a phantom layer, which are both monolayer unit-cells. The phantom layer is placed between the fixed layer at the tube-end and the rest of the SWNT and control by the Langevin equation. Debye temperature of diamond was chosen as the damping parameter of the Langevin equation. The formulation aims to damp the phonons traveling into the phantom layer and hence to prevent the phonons from being reflected at the tube ends. Therefore, ideally, a phantom thermostat models isothermal layers with sufficient length.

For SWNTs, the simulations using the phantom technique was validated by performing an additional set of simulations adopting standard Nose-Hoover (NH) thermostats $^{20,21)}$. A straightforward application of $\mathrm{NH}$ thermostat without any virtual dynamics makes the method simple and robust, though it is more expensive than the previous method. The thermostats have two tuning parameters; the length of the temperature controlled $L_{c}$ and the relaxation time $\tau$. Fig. 2 shows the temperature profiles obtained by using the $\mathrm{NH}$ thermostat for various values of $L_{c}$, where distinct TBRs can be observed as temperature jumps. For instance, in the case of $L_{c}=0.01 \mathrm{~L}$, temperature jumps at the interfaces account for about $50 \%$ of the total temperature difference applied at both tube-ends.

In order to minimize the TBRs, parameters $L_{c}$ and $\tau$ were tuned. Influences of $L_{c}$ and $\tau$ on the key thermal properties are described in Fig. 2-4. Elongation of $L_{c}$ permits larger wavelength phonon modes and hence attenuates the discrepancy of phonon spectra between temperature-controlled part and the rest of the nanotube. This can be seen in the $L_{c}$-dependence of temperature profiles (Fig. 2), where the shorter $L_{c}$ is, the larger TBRs are. More detail views are given in Fig. 3(a-c) which show $L_{c}$-dependences of the temperature gradient, heat flux and thermal conductivity. Both the temperature gradient and heat flux increased with $L_{c}$ and eventually saturated at the upper limit $L_{c} / L \sim 1$, independently of $\tau$. The corresponding trend of $\lambda$ is similar except for the value for $L_{c} / L=0.01$. Therefore, considering the computational cost, we approved $L_{c}=0.5 L$ as an 
optimal value.

As for the relaxation time, longer $\tau$ is expected to give the temperature-controlled layers more time to adjust the spectrum to that of the rest of the SWNT. The variation of TBRs with respect to $\tau$ is shown in Fig. 4 for $L_{c}=0.5 L$. The figure shows the sum of TBRs on the hot and cold sides $R$ for various nanotube lengths, within the parameter bounds ( $40 \mathrm{fs}<\tau<4 \mathrm{~ns}$ ) beyond which the quasi-linear temperature profile is significantly disturbed. Beyond the lower bound, the phonon spectra of temperature-controlled layers and the rest of the SWNT were found to exhibit severe mismatching. On the other hand, beyond the upper bound, the data hardly converged. The figure shows that $R$ takes a minimum value for a critical relaxation time $\tau_{c r}$. On varying $L$ from $25 \mathrm{~nm}$ to $201 \mathrm{~nm}, \tau_{c r}$ exhibits a moderate variation between $400 \mathrm{fs}$ and $40 \mathrm{ps}$. On considering the observed trend that $\tau_{c r}$ increases with $L$, we take $\tau=40 \mathrm{ps}$ as the optimal value. Note that an order difference in $\tau$ may result in approximately $10 \%$ difference in thermal conductivity.

\section{Length and Diameter Effects on SWNT Thermal Conductivity}

Figure 5 shows length effect on thermal conductivity of SWNTs for a range of $L$ up to $1.6 \mu \mathrm{m}$. It can be seen that differences between the values obtained for $(5,5) \mathrm{SWNTs}$ with the phantom technique (filled circles) and $\mathrm{NH}$ thermostats (open circles) are negligible. The overall trend of the slope $(\partial \lambda / \partial L)$ clearly indicates the gradual transition from strongly ballistic to diffusive-ballistic phonon transport. When all the phonons experience ballistic phonon transport, $\lambda$ is proportional to $L$ (constant thermal conductance). The asymptotic match of the gradients of the thermal conductivity profiles to that of the dashed line suggests dominant ballistic phonon transport at the small $L$ limits. Note, on considering the significant phonon population in a range of phonon branches at room temperature, we expect contributions to the heat conduction not only from ballistic transport of acoustic phonon modes but also from that of various optical phonon modes in the small $L$ regime. This is consistent with the results of MD realization of non-Fourier heat conduction in tens of nanometers long SWNTs, where the ballistic transport of collective optical phonons was observed to play an important role. ${ }^{18)}$ The gradient $\partial \lambda / \partial L$ gradually decreases as $L$ increases since phonon mean free paths gradually become shorter relatively to $L$, i.e. diffusive phonon transport is gradually enhanced with respect to ballistic phonon transport. For both systems, thermal conductivity did not converge in the range of $L$ explored in the current work up to about a micrometer. Furthermore, in connection with the discussion on the divergence of thermal conductivity ${ }^{11,12)}$, one could fit power-law functions to the obtained profiles. As denoted in Fig. 5, by fitting power laws in $L>100 \mathrm{~nm}$, we obtain exponents of 0.19 and 0.33 for $(5,5)$ and $(3,3)$ SWNTs, respectively. The power-law length-dependence of thermal conductivity could be explained by the weak 3-phonon scattering of long 
wavelength phonons compromised by the higher order processes ${ }^{12)}$, and/or the long time heat current correlations in low dimensions ${ }^{22}$.

Let us now examine the diameter $(d)$ dependence of SWNT thermal conductivity. For small $L$, where the phonon transport is dominantly ballistic, the thermal conductivity exhibits minor dependence on the diameter. The diameter-independence for small $L$ is consistent with the above discussion that ballistic phonon transport is dominant in this regime. At the ballistic phonon transport limit, where all the populated phonons experience ballistic transport, thermal conductance is proportional to the number of atoms per unit cell, i.e. the diameter, if we ignore the variation of the linear phonon transport property (dispersion relations) due to the changes in the unit-cell size and the curvature. This means, with the current definition of $A=\pi b d$, that the thermal conductivity is independent of the diameter. As $L$ increases, the diameter dependence becomes noticeable, where thermal conductivity profiles of $(3,3)$ and $(5,5)$ SWNTs deviate from each other beyond $L \sim 100 \mathrm{~nm}$. Current results show that, in the large $L$ regime, thermal conductivity is larger i.e. the diffusion effect is smaller for SWNTs with smaller $d$. The trend is consistent with the above mentioned candidates of origin of the power-law length dependence. The exponent of the thermal conductivity divergence due to the long wave-length phonons increases as $d$ decreases ${ }^{13)}$ because of the variation in the phonon density of states. Similarly, the long time correlation ${ }^{22)}$ is also expected to increase as $d$ decreases due to the reduction of number of phonon-channels per length. The exact mechanism of the power-law length-dependence can not be identified from the present diameter-dependence of the exponent. Detailed examination of heat flux autocorrelation function in the equilibrium framework would be needed to discuss this aspect further.

\section{Conclusions}

Non-equilibrium MD simulations were conducted to investigate the heat conduction of SWNTs at room temperature. The length and diameter effects on the thermal conductivity were quantified in a range of $L$ and for two different diameters. The gradual transition from nearly pure ballistic phonon transport to diffusive-ballistic phonon transport was clearly observed. In the small $L$ regime with strong ballistic transport, there is a significant contribution to the heat conduction from a range of optical phonons. Consistent picture of ballistic phonon transport was obtained from the diameter dependence, where thermal conductivity is diameter-invariant for small $L$. In the regime of $L$ with significant diffusive phonon transport, power-law length dependence was identified whose exponent increases with reducing diameter. In this regime, thermal conductivity of an SWNT shows power-law length-dependence does not converge even with a micrometer tube-length. 


\section{Acknowledgements}

This work is supported in part by Grants-in-Aid for Scientific Research \#19206024, \#19054003, \#19051016, \#19860022.

\section{References}

1. R. Saito, G. Dresselhaus and M. S. Dresselhaus, Physical Properties of Carbon Nanotubes, Imperial College Press, London (1998).

2. S. Berber, Y-K. Kwon and D. Tomanek, Phys. Rev. Lett. 84, 4613 (2000).

3. J. Hone, M.C. Llaguno, M. J. Biercuk, A.T. Johnson, B. Batlogg, Z. Benes, J. E. Fischer, Appl. Phys. A 74, 339 (2002).

4. P. Kim, L. Shi, A. Majumdar, and P. L. McEuen, Phys. Rev. Lett. 87, 215502 (2001).

5. M. Fujii, X. Zhang, H. Xie, H. Ago, K. Takahashi, T. Ikuta, H. Abe and T. Shimizu, Phys. Rev. Lett. 95, 065502 (2005).

6. C. Yu, L. Shi, Z. Yao, D. Li and A. Majumdar, Nano Lett. 5, 1842 (2005).

7. E. Pop, D. Mann, Q. Wang, K. Goodson and H. Dai 6, 96 (2006).

8. G. Chen, Nanoscale Energy Transport and Conversion, Oxford University Press, New York (2005).

9. S. Maruyama, Physica B, 323, 193 (2002).

10. S. Maruyama, Micro. Therm. Eng., 7, 41 (2003).

11. R. Livi and S. Lepri, Nature, 421, 327 (2003).

12. N. Mingo and D. A. Broido, Nano Lett. 5, 1221 (2005).

13. J. Wang and J-S Wang, Appl. Phys. Lett. 88, 111909 (2006).

14. D. W Brenner, Phys. Rev. B, 42, 9458 (1990).

15. Y. Yamaguchi and S. Maruyama, S., Chem. Phys. Lett., 286, 336 (1998).

16. J. Hone, M. Whitney, C. Piskoti, A. Zettl, Phys. Rev. B. 59, R2514 (1999).

17. T. Yamamoto, S. Watanabe, K. Watanabe, Phys. Rev. Lett. 92, 075502 (2004).

18. J. Shiomi and S. Maruyama, Phys. Rev. B 73, 205420 (2006).

19. G. D. Mahan and G. S. Jeon, Phys. Rev. B, 70, 075405 (2004).

20. S. Nose, J. Chem. Phys., 81 (1), 511 (1984).

21. W. G. Hoover, Phys. Rev. A, 31, 1695 (1985).

22. S. Lepri, R. Livi and A. Politi, Phys. Report, 377, 1, (2003). 


\section{Figure captions}

Fig. 1 (a) Discrete phonon dispersion relations and (b) phonon density of states (DOS) of a $25 \mathrm{~nm}$-long $(5,5)$ SWNT. The dispersion relations were obtained by computing the phonon energy spectral density from MD simulations ${ }^{18)}$. Wavevector $k$-space is normalized by the Brillouin-zone width, $\pi / a$. In the current case with an armchair SWNT, $a=\sqrt{3} A_{c-c}$, where $A_{c-c}$ is the interatomic distance. The focused view (c) shows the phonon dispersion in the low frequency region for clarity.

Fig. 2 Influence of the length of the layers controlled by Nose-Hoover thermostat $\left(L_{c}\right)$ on the temperature profile $(L=25 \mathrm{~nm})$.

Fig. 3 Influence of $L_{c} / L$ and relaxation time of Nose-Hoover thermostat on the axial temperature gradient $d T / d z$, heat flux through the system $Q$ and thermal conductivity $\lambda . L=25 \mathrm{~nm}$.

Fig. 4 Influence of the relaxation time of Nose-Hoover thermostat on the sum of TBRs on the hot and cold sides for different values of $L\left(L_{c}=0.5 L\right)$. The Dashed line marks $\tau=40$ ps.

Fig. 5 Length dependences of SWNT thermal conductivity with two different diameters. $\lambda_{\mathrm{p}}$ and $\lambda_{\mathrm{NH}}$ denote the values obtained by using phantom and Nose-Hoover thermostats, respectively. The error bars are based on the fitting residuals in the thermal conductivity calculations. Thermal conductivity profiles of $(3,3)$ and $(5,5)$ SWNTs in $\mathrm{L}>100 \mathrm{~nm}$ were fitted to power laws. Dashed line shows $\lambda \propto L$ with an arbitrary slope. 


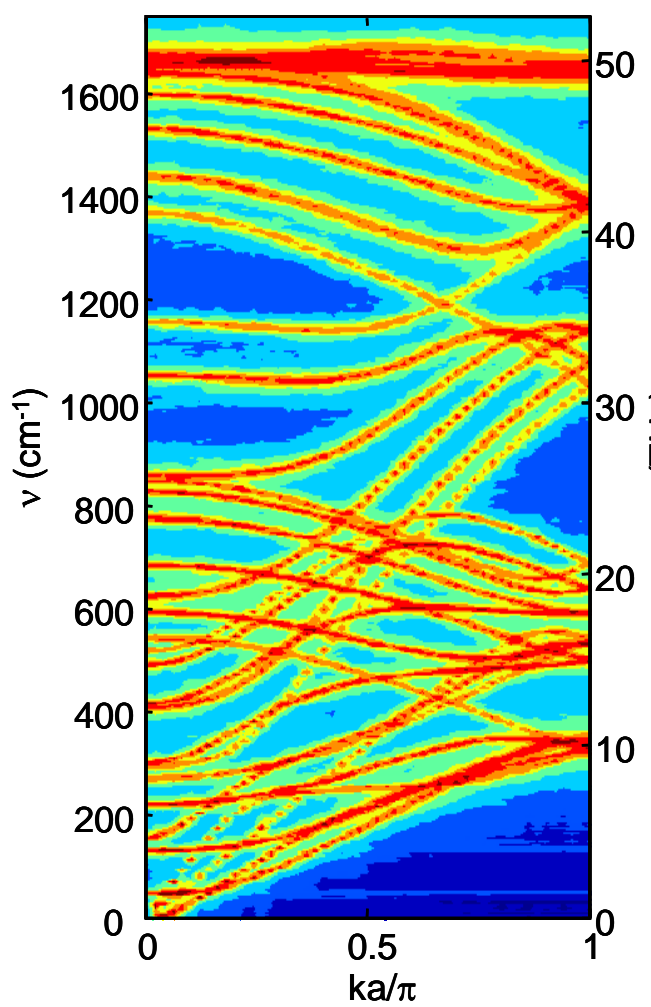

(a) Phonon dispersion

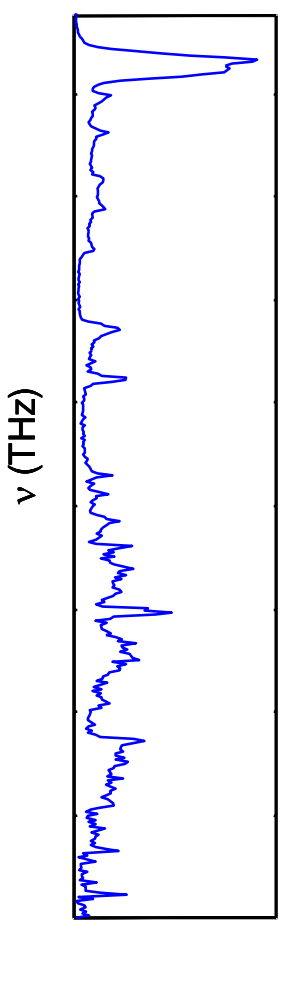

(b) Phonon DOS

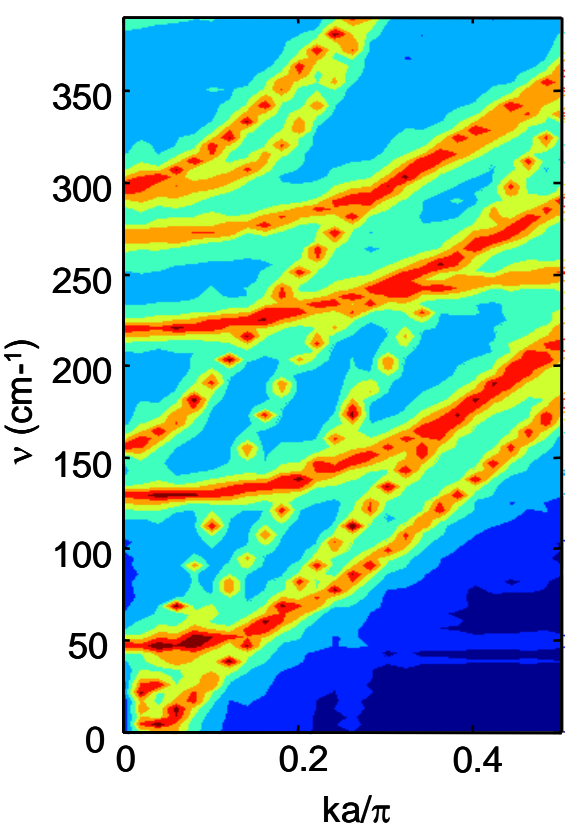

(c) Focused view of (a)

FIG. 1 J. Shiomi and S. Maruyama 


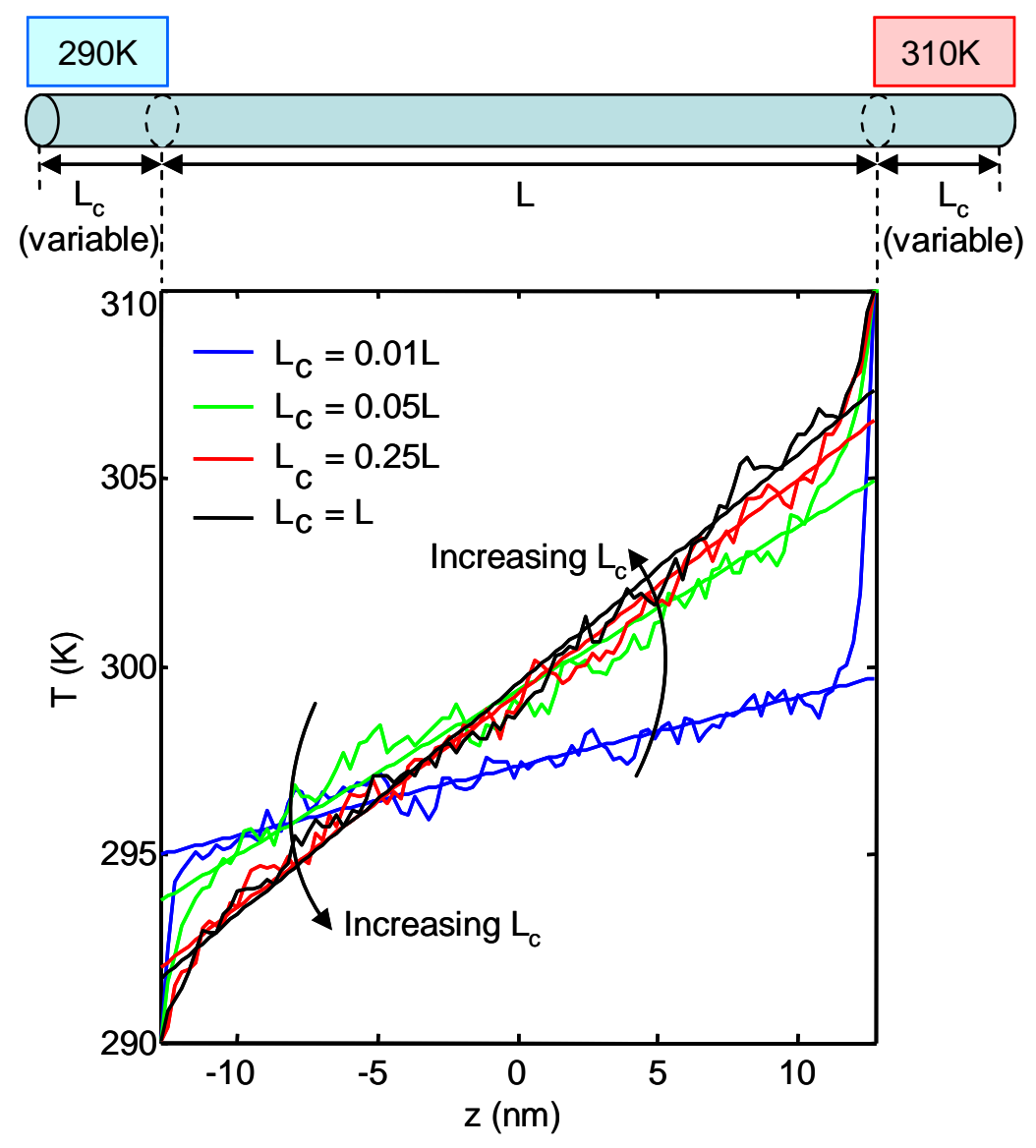

FIG. 2 J. Shiomi and S. Maruyama 


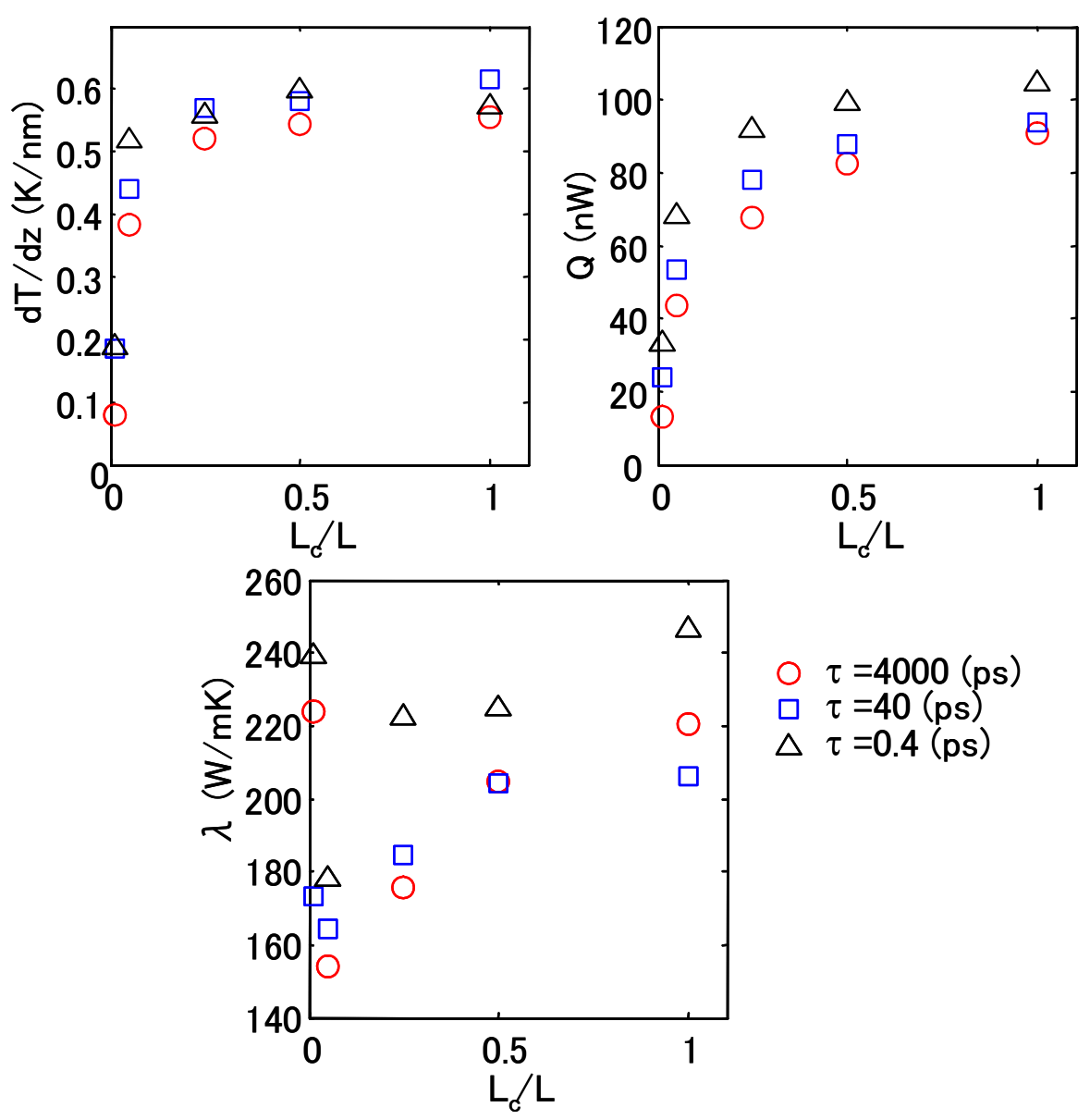

FIG. 3 J. Shiomi and S. Maruyama 


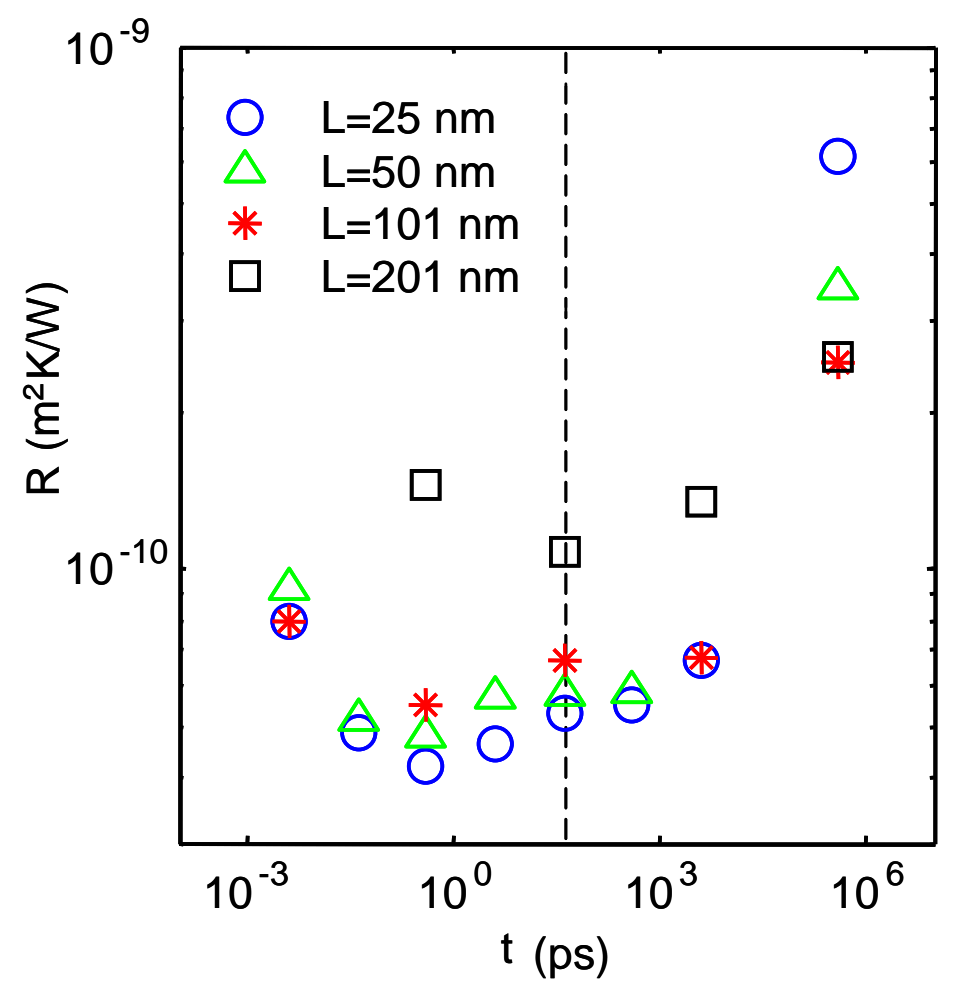

FIG. 4 J. Shiomi and S. Maruyama 


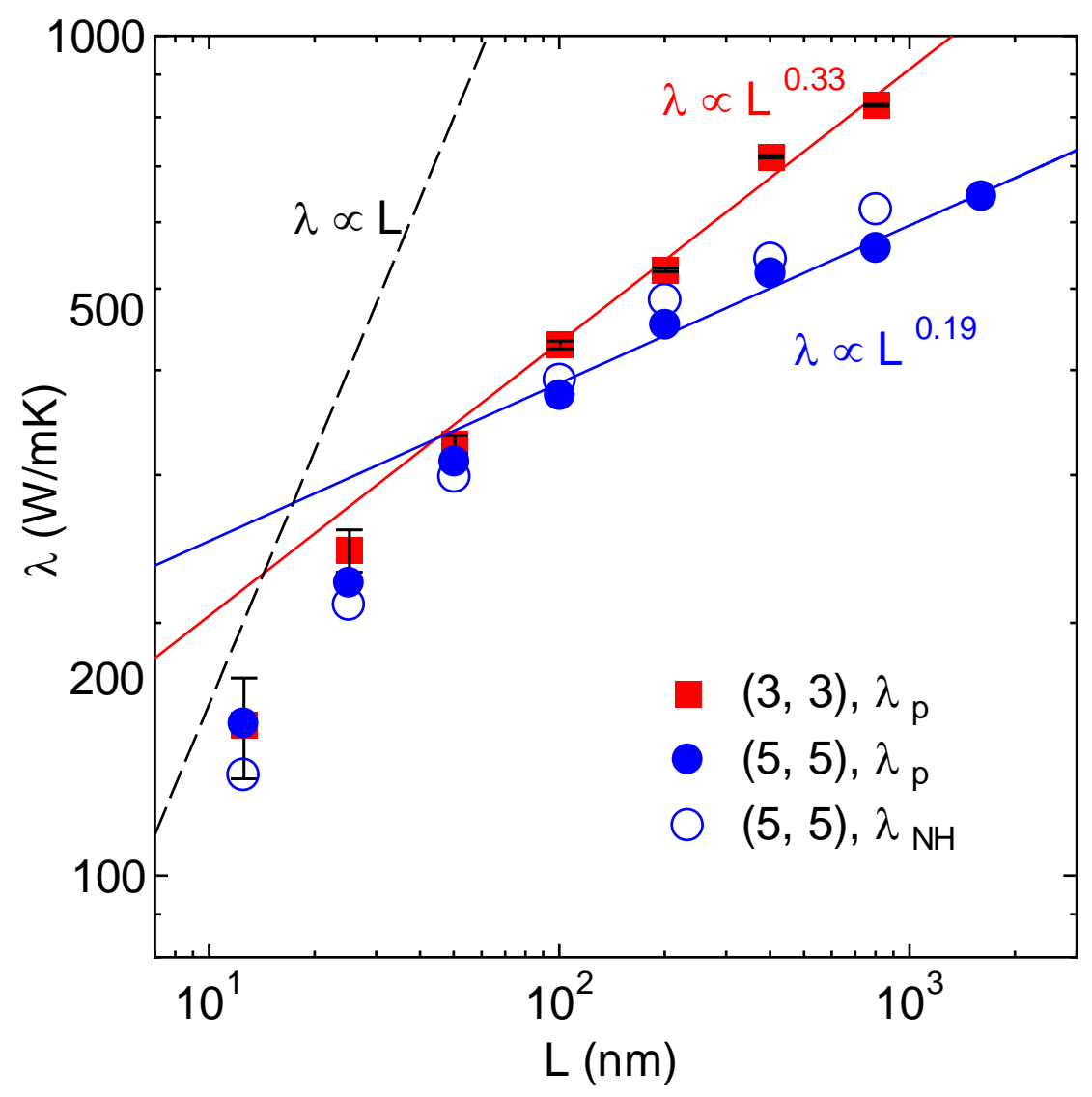

FIG. 5 J. Shiomi and S. Maruyama 